\title{
Identifying potential novel insights for COVID-19 pathogenesis and therapeutics using an integrated bioinformatics analysis of host transcriptome
}

\section{Salem El-aarag}

Bioinformatics Department, Genetic Engineering and Biotechnology Research Institute (GEBRI), University of Sadat City, Egypt.

Amal Mahmoud ( $\square$ amhsalem@iau.edu.sa )

Department of Biology, College of Science, Imam Abdulrahman Bin Faisal University, Dammam, Saudi Arabia https://orcid.org/0000-0001-7691-3656

Mahmoud ElHefnawi ( $\nabla$ mahef@aucegypt.edu )

Biomedical informatics and chemoinformatics group, Informatics and systems department, National Research Center, Egypt. https://orcid.org/0000-0003-3896-6911

\section{Research Article}

Keywords: COVID-19, Systems biology, Differentially expressed genes, Functional module analysis, Protein-protein interaction network, Enrichment analysis, host transcriptome and drug repurposing.

Posted Date: April 15th, 2021

DOl: https://doi.org/10.21203/rs.3.rs-406292/v1

License: (9) This work is licensed under a Creative Commons Attribution 4.0 International License. Read Full License

Version of Record: A version of this preprint was published at International Journal of Biological Macromolecules on November 1st, 2021. See the published version at https://doi.org/10.1016/j.jibiomac.2021.11.124. 
Identifying potential novel insights for COVID-19 pathogenesis and therapeutics using an integrated bioinformatics analysis of host transcriptome

Salem A. El-aarag ${ }^{1}$, Amal Mahmoud², and Mahmoud ElHefnawi ${ }^{3}$

${ }^{1}$ Bioinformatics Department, Genetic Engineering and Biotechnology Research Institute (GEBRI), University of Sadat City, Egypt.

${ }^{2}$ Department of Biology, College of Science, Imam Abdulrahman Bin Faisal University, Dammam, Saudi Arabia

${ }^{3}$ Biomedical informatics and chemoinformatics group, Informatics and systems department, National Research Center, Egypt.

E-mail addresses:

Salem El-aarag: salim.alaarag@yahoo.com

Amal Mahmoud: amal.mahmoud@gebri.usc.edu.eg

Mahmoud ElHefnawi: mahef@aucegypt.edu

*Corresponding Author Email: amhsalem@iau.edu.sa (A.M.) and mahef@aucegypt.edu. 


\begin{abstract}
The molecular mechanisms underlying the pathogenesis of COVID-19 has not been fully discovered. This study aims to decipher potentially hidden parts of the pathogenesis of COVID-19, potential novel drug targets, and to identify potential drug candidates. Two gene expression profiles (GSE147507GSE153970) were analyzed and overlapping differentially expressed genes (DEGs) were selected for which top enriched transcription factors and kinases were identified and pathway analysis was performed. Protein-protein interaction (PPI) of DEGs was constructed, hub genes were identified and module analysis was also performed. DGIdb database was used to identify drugs for the potential targets (hub genes and the most enriched transcription factors and kinases for DEGs). A drug-potential target network was constructed and drugs are ranked according to the degree. L1000FDW web-based utility was used to identify drugs that can reverse transcriptional profiles of COVID-19. We identified drugs currently in clinical trials and novel potential 8 drugs. Besides the well-known pathogenic pathways, It was found that axon guidance is a potential pathogenic pathway. Sema7A, which may exacerbate hypercytokinemia, is considered a potential novel drug target. Another potential novel pathway is related to TINF2 overexpression which may induce potential telomere dysfunction and hence DNA damage that may exacerbate lung fibrosis.
\end{abstract}

Keywords: COVID-19, Systems biology, Differentially expressed genes, Functional module analysis, Protein-protein interaction network, Enrichment analysis, host transcriptome and drug repurposing. 


\section{Background}

Severe acute respiratory syndrome coronavirus-2 (SARS-CoV-2) is a member of the Coronaviridae family, isolated at the end of 2019. SARS-CoV-2 pandemic has about 3.06\% mortality rate according to WHO. Nearly, the majority of COVID-19 patients, about $80 \%$, have mild symptoms. Nearly $20 \%$ of cases are severe and develop severe respiratory distress syndrome [1].

Several studies reported that dysregulated immune response (hypercytokinemia) is associated with severe cases [2-6]. Uncontrolled host immune response may be responsible for the fatal outcome rather than unabated viral replication as it was observed that fatal cases had a low viral load [7] so that understanding the host response to SARS-CoV-2 is critical to find potential therapeutic targets for severe COVID-19 disease [7].

In this study, we tried to understand the underlying pathological pathways related to SARS-CoV-2 infection and identify potential drug targets and drugs that can be used for combating SARS-CoV-2induced injuries. Two RNA-seq datasets (GSE147507-GSE153970) were analyzed and DEGs were identified based on the two datasets. Gene ontology and the Kyoto encyclopedia of genes and genomes pathway enrichment analysis for DEGs were performed. Also, top enriched transcription factors and kinases for DEGs were identified. Protein-protein interaction (PPI) of DEGs was constructed and hub genes were identified. DGIdb database was used to identify drugs for the potential targets (hub genes and the most enriched transcription factors and kinases for DEGs). A drug-potential target network was constructed and drugs were ranked according to the degree. L1000FDW was searched for drugs that can reverse transcriptional profiles of COVID-19. PPI interaction Network of DEGs was expanded and GO biological process enrichment analysis and KEGG pathway analysis of the top eight modules were performed. We identified drugs already in use in clinical trials against COVID-19 disease as well as new potential drugs. We also identified new potential drug targets and new 
pathogenic pathways for COVID-19 disease. .

\section{Methods}

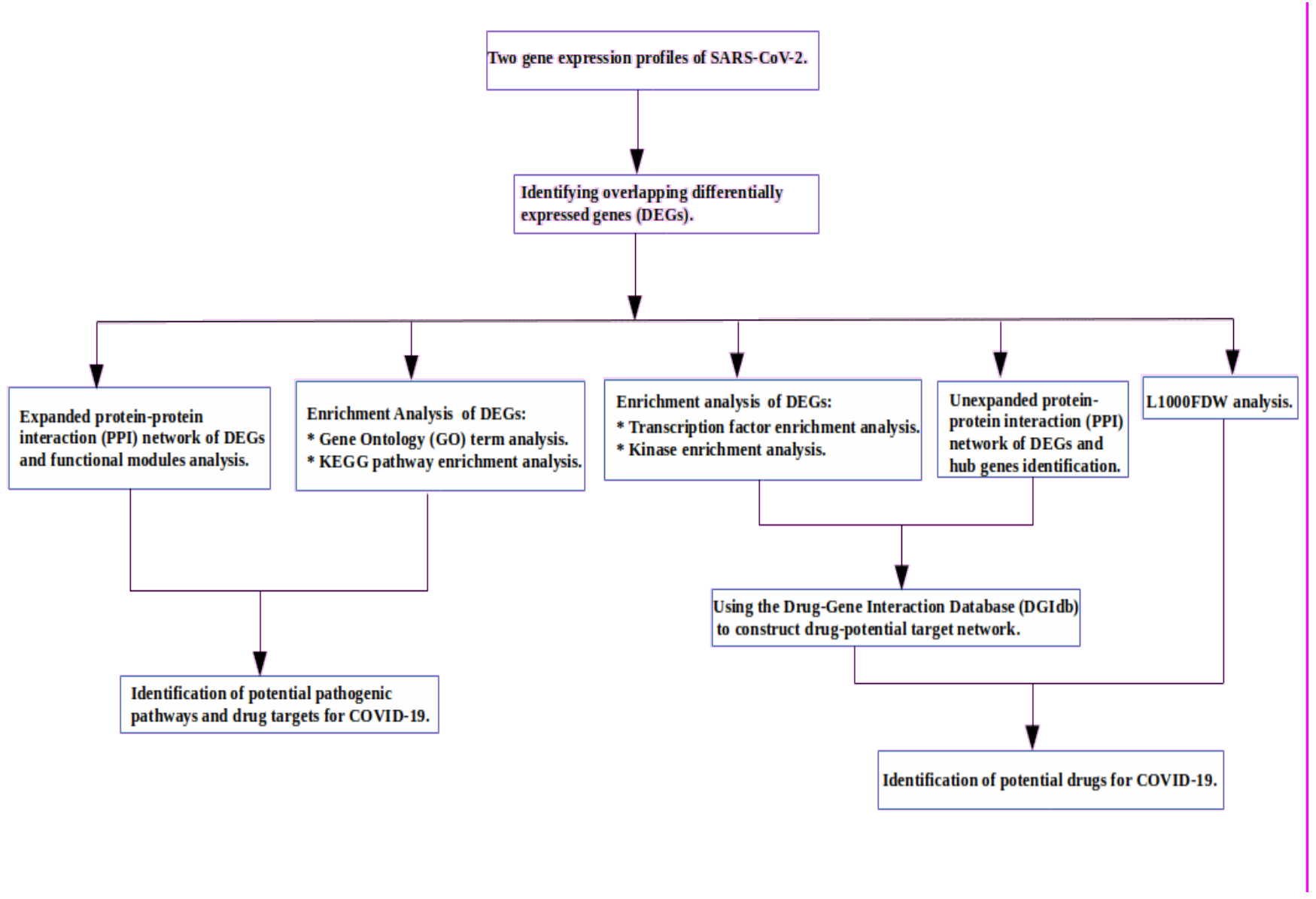

In Fig. 1, we present a Flow chart of our integrated novel bioinformatics approach to identify potential pathogenic pathways, drug targets, and drug candidates for COVID-19.

\section{1- Datasets}

Two gene expression profiles (GSE147507- GSE15397) were obtained from the NCBI Gene Expression Omnibus (GEO) database (http://www.ncbi.nlm.nih.gov/geo/). The first dataset is stranded RNA-seq data from mock-treated and SARS-CoV-2 (USA-WA1/2020 at MOI of 2 for 24 h.)-infected primary human lung epithelium (NHBE). Each group included three independent samples. Each sample has four runs. The second dataset is unstranded RNA-seq data from mock-treated and SARS- 
CoV-2 (USA-WA1/2020 at MOI of 0.5 for 48 h)-infected Normal Airway Epithelial cells (NAE). Each group included three independent samples. Each sample has two runs.

\section{2- Identifying DEGs}

We used Galaxy [https://usegalaxy.org/] as a platform for RNA-seq analysis. First, raw reads were subjected to quality control analysis using FastQC [11] before and after using trimmomatic tool [12] which was run with its default options. Trimmed reads were aligned against the reference genome (hg19) using HISAT2 [13]. All aligned runs for the same sample were merged using MergeSamFiles [14]. Aligned reads that overlap features in a GFF file (gencode.v19.annotation.gtf.gz) were count using htseq-count [15]. DESeq2 [16] was used to determine differentially expressed features count tables. Differentially expressed genes (DEGs) were considered using (q. val < 0.05).

\section{3- Pathway and GO enrichment Analysis of DEGs}

Gene Ontology (GO) term analysis and KEGG pathway enrichment analysis of DEGs were performed using STRING (https://string-db.org/). Transcription factors enrichment analysis and kinases enrichment analysis of DEGs were performed using ChEA3 [17] and KEA3 (https://amp.pharm.mssm.edu/kea3/) respectively using the integrated mean rank method.

\section{Hub genes identification}

PPI interaction network was obtained by submitting DEGs to the STRING and analysed by Cytoscape software (version 3.8.1) [18]. Hub genes were selected based on the intersection of three methods; CytoNCA [19] using two centrality methods (subgraph-degree) and Cytohubba [20].

\section{Identifying potential drugs}

\subsection{Drug-potential target network}

Using the Drug-Gene Interaction Database (DGIdb; http://www.dgidb.org/search_interactions), drugs were selected based on the hub genes and the most enriched transcription factors and kinases for DEGs that served as promising drug targets [10]. Cytoscape software was used to draw the drug- 
potential target network. Drugs are ranked according to the degree using Cyto-NCA. Only FDA approved drugs were selected. We neglected drugs that are not for internal use like topical cream and eye drop.

\subsection{L1000FDW analysis}

L1000FDW was used to identify potential drugs which can reverse the transcriptional profiles of COVID-19 [21]. DEGs were used as input. Only FDA approved drugs were selected.

\section{Functional modules analysis}

PPI interaction Network of DEGs was expanded using STRING and analysed by Cytoscape software (version 3.8.1) [18]. Functional modules were identified via the MCODE using ClusterViz [22-23] with the default parameters. GO biological process enrichment analysis and KEGG pathway analysis of genes in each functional modules were done using STRING.

\section{Results}

\section{DEGs}

We identified 422 DEGs between SARS-CoV-2 infected NHBE cells and mock-treated NHBE cells. and 1829 DEGs between SARS-CoV-2 infected NAE and mock-treated cells. 187 overlapping genes (48 downregulated and 139 upregulated genes) were selected using VENNY 2.1 (https://bioinfogp.cnb.csic.es/tools/venny/index.html) [24] (Fig. 2 and Table S1).
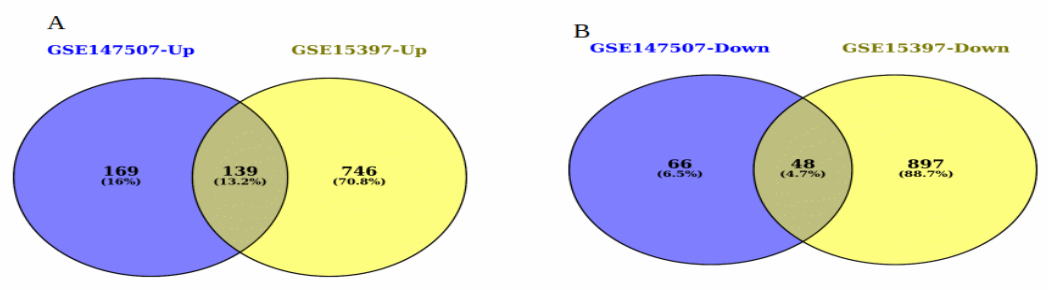

Fig. 2. Venn diagram for overlapping differentially expressed genes (DEGs) based on two datasets. 
(A) the intersection of upregulated DEGs and (B) the intersection of downregulated DEGs dentified from the two datasets, namely, GSE147507 and GSE15397.

\section{Pathway and GO enrichment Analysis of DEGs}

KEGG pathway analysis of DEGs was enriched to IL-17 signaling pathway, TNF signaling pathway, cytokine-cytokine receptor interaction, NF-kappa B signaling pathway, rheumatoid arthritis, and NOD-like receptor signaling pathway (Fig. 3A) whereas they were significantly involved in the immune process, inflammatory response, response to an external stimulus, response to biotic stimulus, defence response, and response to cytokine (Fig. 3B), highly enriched to cytokine activity, molecular function regulator, molecular function regulator, protein binding and cytokine receptor binding (Fig. 3C), and correlated to extracellular space, secretory granule, cytoplasmic vesicle, and vesicle (Fig. 3D).

RELB, ARNTL2, GRHL3, PLSCR1, SNAI, CSRNP1, ZNF750, OVOL1, FOSL1, and ZNF267 were the top-ranked transcription factors for DEGs whereas EGFR, RIPK1, CHUK, IKBKB, SRC, TBK1, PAK1, IRAK2, FYN,8, and LYN were the most enriched kinases (Table. 1). We considered the top enriched transcription factors and kinases for DEGs as potential drug targets [10].

\section{Hub genes identification}

The PPI network included 186 nodes, 654 edges with an average node degree of 7.03 and avg. local clustering coefficient of 0.49 , and its PPI enrichment p-value was $<1.0 \mathrm{e}-16$ (Fig. 4). We used three methods for identifying hub genes; CytoNCA using two centrality methods (subgraph-degree) and Cytohubba. The top 20 hub genes were identified from each method. 18 overlapping hub genes (IL6, CXCL8, CXCL1, CCL20, CXCL2, CXCL3, IL1B, IL1A, MMP9, CXCL5, CSF3, ICAM1, IL1RN, CSF2, PTGS2, SAA1, NFKB1, and TNFAIP3) were selected based on the intersection of the three methods (Table. 2). The PPI network of the hub genes was constructed using STRING (Fig. 5) and consisted of 18 nodes and 142 edges, while its average local clustering coefficient was 0.943 , and its 
PPI enrichment P-value was lower than 1.0e - 16. This showed that these hub genes highly interact with each other.
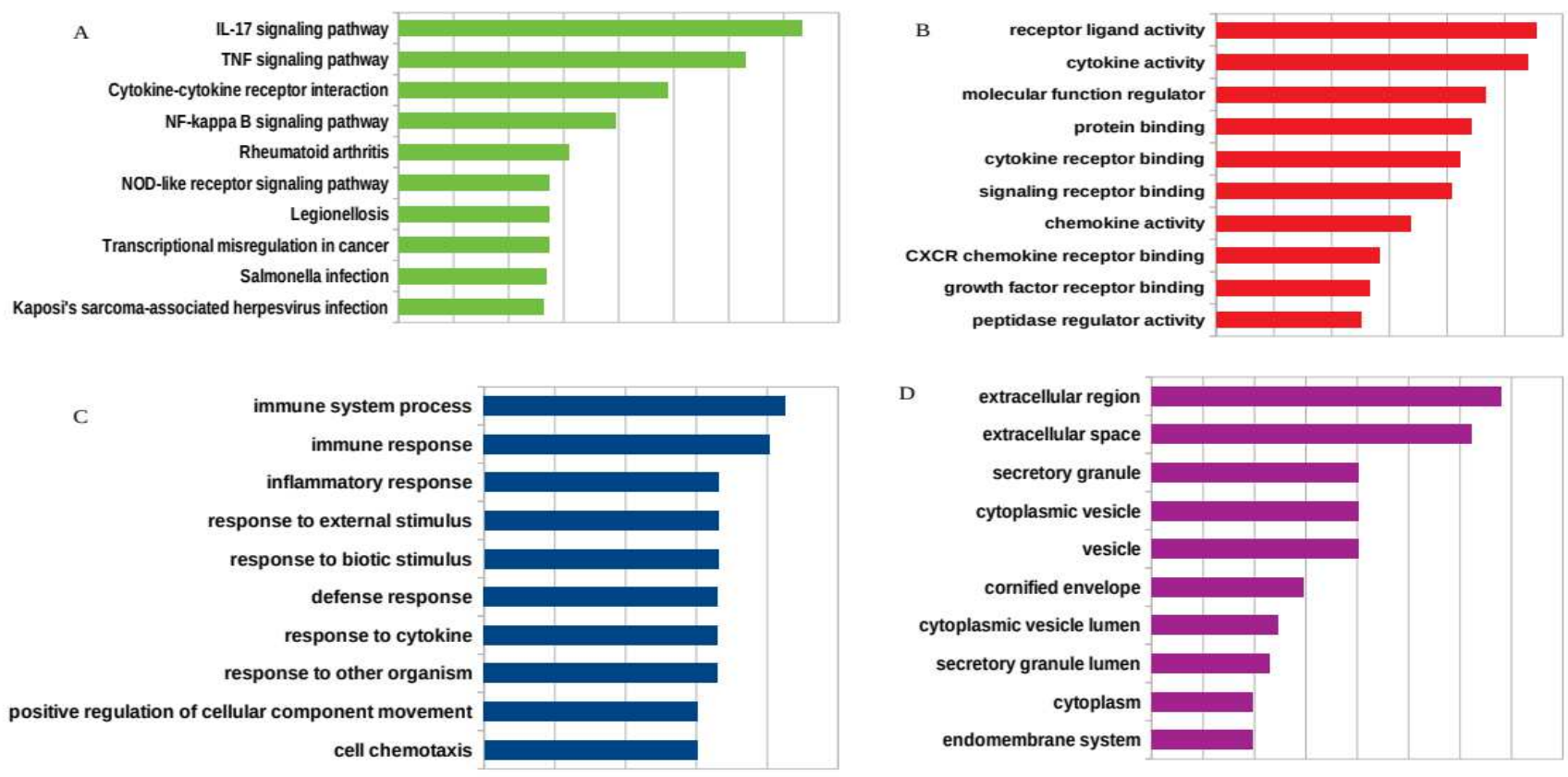

Fig. 3. Enrichment analysis of DEGs. A. KEGG pathway enrichment analysis of DEGs. B. Biological function enrichment analysis of DEGs. C. Biological process enrichment analysis of DEGs. D. Biological component enrichment analysis of DE.

Table 1. Top enriched transcription factors and kinases for DEGs.

\begin{tabular}{|c|c|c|}
\hline Rank & Transcription factors & Kinases \\
\hline 1 & RELB & EGFR \\
\hline 2 & ARNTL2 & RIPK1 \\
\hline 3 & GRHL3 & CHUK \\
\hline 4 & PLSCR1 & IKBKB \\
\hline 5 & SNAI1 & SRC \\
\hline 6 & CSRNP1 & TBK1 \\
\hline 7 & ZNF750 & PAK1 \\
\hline 8 & OVOL1 & IRAK2 \\
\hline
\end{tabular}




\begin{tabular}{|c|c|c|}
\hline 9 & FOSL1 & FYN \\
\hline 10 & ZNF267 & LYN \\
\hline
\end{tabular}

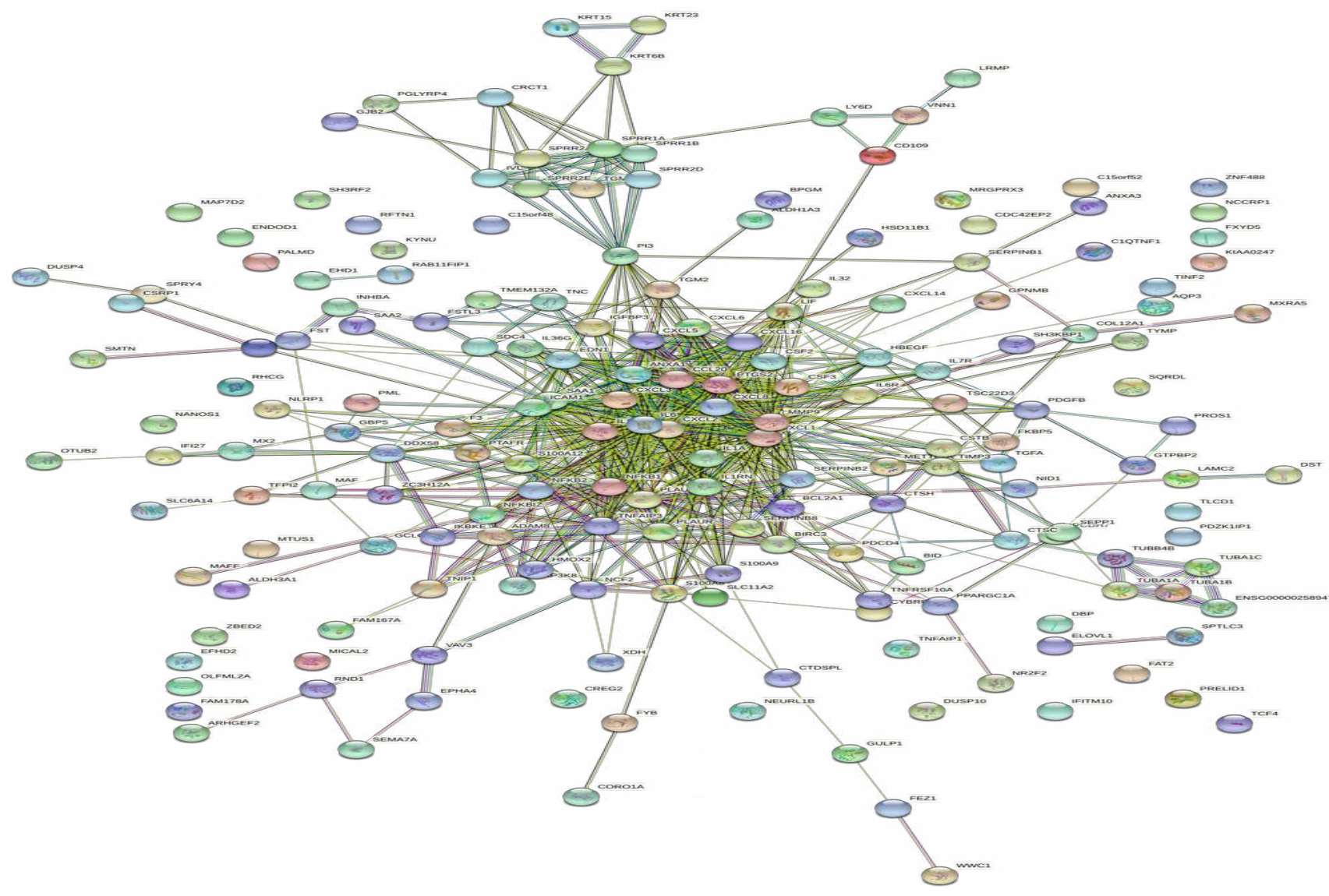

Figure 4. Unexpanded PPI network of DEGs using STRING.

Drug-potential target network

Potential targets consisted of 18 hub genes, top 10 enriched kinases and transcription factors for DEGs were searched for promising drugs using DGIdb. We didn't find drugs for all potential transcription factors except FOSL1. However, all hub genes and potential kinases have drugs except CXCL1, CCL20, CXCL3, CXCL5 and IRAK2. We included only two FDA unapproved botanical derived drugs. 215 potential drugs were obtained (Supplementary data 2). We concentrated on drugs with degree $>=3$ as Fostamatinib, Entrectinib, Ponatinib, Dasatinib, Bosutinib, Ibrutinib, and Acalabrutinib were the top interactive drugs that act on multi-drug targets (Table 3, Fig. 6). 
Table 2. Identified hub genes by CytoNCA using two centrality methods (degree and subgraph) and Cytohubba.

\begin{tabular}{|l|c|c|l|l|l|}
\hline Rank & Cytohubba & $\begin{array}{l}\text { CytoNCA- } \\
\text { Degree }\end{array}$ & $\begin{array}{l}\text { CytoNCA- } \\
\text { Subgraph }\end{array}$ & $\begin{array}{l}\text { Common hub } \\
\text { genes }\end{array}$ & Description \\
\hline 1 & IL6 & IL6 & IL6 & IL6 & Interleukin 6 (IL6) \\
\hline 2 & CXCL8 & CXCL8 & CXCL8 & CXCL8 & C-X-C motif chemokine ligand 8 (CXCL8) \\
\hline 3 & CXCL1 & IL1B & IL1B & CXCL1 & C-X-C motif chemokine ligand 1 (CXCL1) \\
\hline 4 & CCL20 & MMP9 & MMP9 & CCL20 & C-C motif chemokine ligand 20 (CCL20) \\
\hline 5 & CXCL2 & CXCL1 & CXCL1 & CXCL2 & C-X-C motif chemokine ligand 2 (CXCL2) \\
\hline 6 & CXCL3 & PTGS2 & PTGS2 & CXCL3 & C-X-C motif chemokine ligand 3 (CXCL3) \\
\hline 7 & IL1B & ICAM1 & ICAM1 & IL1B & Interleukin 1 Beta (IL1B) \\
\hline 8 & IL1A & CSF2 & CCL20 & IL1A & Interleukin 1 Alpha (IL1A) \\
\hline 9 & MMP9 & CCL20 & CSF2 & MMP9 & Matrix Metallopeptidase 9 (MMP9) \\
\hline 10 & CXCL5 & IL1A & IL1A & CXCL5 & C-X-C motif chemokine ligand 5 (CXCL5) \\
\hline 11 & CSF3 & NFKB1 & CXCL2 & CSF3 & Colony Stimulating Factor 3 (CSF3) \\
\hline 12 & ICAM1 & CXCL2 & CXCL3 & ICAM1 & Intercellular Adhesion Molecule 1(ICAM1) \\
\hline 13 & IL1RN & IL1RN & IL1RN & IL1RN & Interleukin 1 Rreceptor Antagonist (IL1RN) \\
\hline 14 & CSF2 & CSF3 & CSF3 & CSF2 & Colony Stimulating Factor 2 (CSF2) \\
\hline 15 & PTGS2 & SAA1 & SAA1 & PTGS2 & Prostaglandin-Endoperoxide Synthase 2 (PTGS2) \\
\hline 16 & SAA1 & CXCL3 & CXCL5 & SAA1 & Serum Amyloid A1 (SAA1) \\
\hline 17 & CXCL6 & TNFAIP3 & NFKB1 & NFKB1 & Nuclear Factor Kappa B Subunit 1(NFKB1) \\
\hline 18 & NFKB1 & PI3 & TNFAIP3 & TNFAIP3 & TNF Alpha Induced Protein 3 (TNFAIP3) \\
\hline 19 & TNFAIP3 & NFKB2 & CXCL6 & & \\
\hline 20 & CXCL16 & CXCL5 & EDN1 & & \\
\hline
\end{tabular}

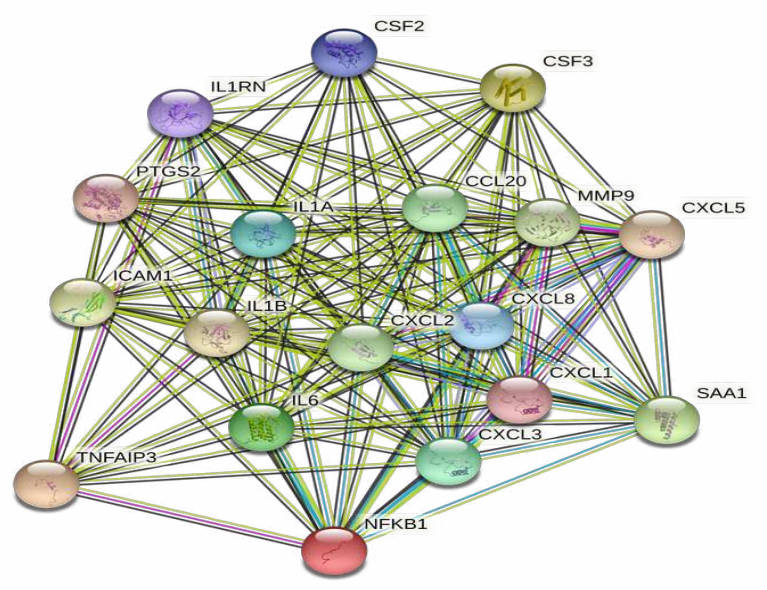

Figure 5. The PPI network of the hub genes using STRING. 
Table 3. Potential drugs with high degree of connectivity identified by CytoNCA from Drug-Potential targets network.

\begin{tabular}{|c|c|c|c|}
\hline Rank & Drugs & Degree & Potential targets (DGIdb) \\
\hline 1 & Fostamatinib & 8 & EGFR-RIPK1-IKBKB-SRC-TBK1-PAK1-FYN-LYN \\
\hline 2 & Entrectinib & 4 & SRC-TBK1-FYN-LYN \\
\hline 3 & Ponatinib & 4 & EGFR-RIPK1-SRC-LYN \\
\hline 4 & Dasatinib & 4 & EGFR-SRC-FYN-LYN \\
\hline 5 & Bosutinib & 4 & EGFR-SRC-FYN-LYN \\
\hline 6 & Ibrutinib & 4 & EGFR-SRC-FYN-LYN \\
\hline 7 & Acalabrutinib & 4 & EGFR-SRC-FYN-LYN \\
\hline 8 & Resveratrol & 4 & IL1B-PTGS2-NFKB1-RIPK1 \\
\hline 9 & Nintedanib & 3 & SRC-FYN-LYN \\
\hline 10 & Vandetanib & 3 & EGFR-SRC-FYN \\
\hline 11 & Gefitinib & 3 & EGFR-IKBKB-LYN \\
\hline 12 & Sulfasalazine & 3 & PTGS2-CHUK-IKBKB \\
\hline 13 & Mesalamine & 3 & PTGS2-CHUK-IKBKB \\
\hline 14 & Curcumin & 3 & MMP9-PTGS2-EGFR \\
\hline 15 & Rilonacept & 3 & IL1B-IL1A-IL1RN \\
\hline 16 & Alteplase & 3 & CXCL2-IL1B-FOSL1 \\
\hline 17 & Aspirin & 3 & CXCL8-IL1B-PTGS2 \\
\hline 18 & Bevacizumab & 3 & CXCL8-MMP9-EGFR \\
\hline 19 & Paclitaxel & 3 & CXCL8-EGFR-SRC \\
\hline 20 & Omeprazole & 3 & CXCL8-IL1B-CSF2 \\
\hline 21 & Naproxen & 3 & CXCL8-PTGS2-SAA1 \\
\hline 22 & Cisplatin & 3 & IL6-EGFR-SRC \\
\hline 23 & Ribavirin & 3 & IL6-EGFR-CXCL8 \\
\hline
\end{tabular}

\section{L1000FDW analysis}

Drugs with low similarity scores may potentially reverse the transcriptional profile of COVID-19 and are potential drugs for COVId-19. Sorafenib, Sirolimus, Selumetinib, Fostamatinib, Dasatinib were the top drugs (Table 4). 


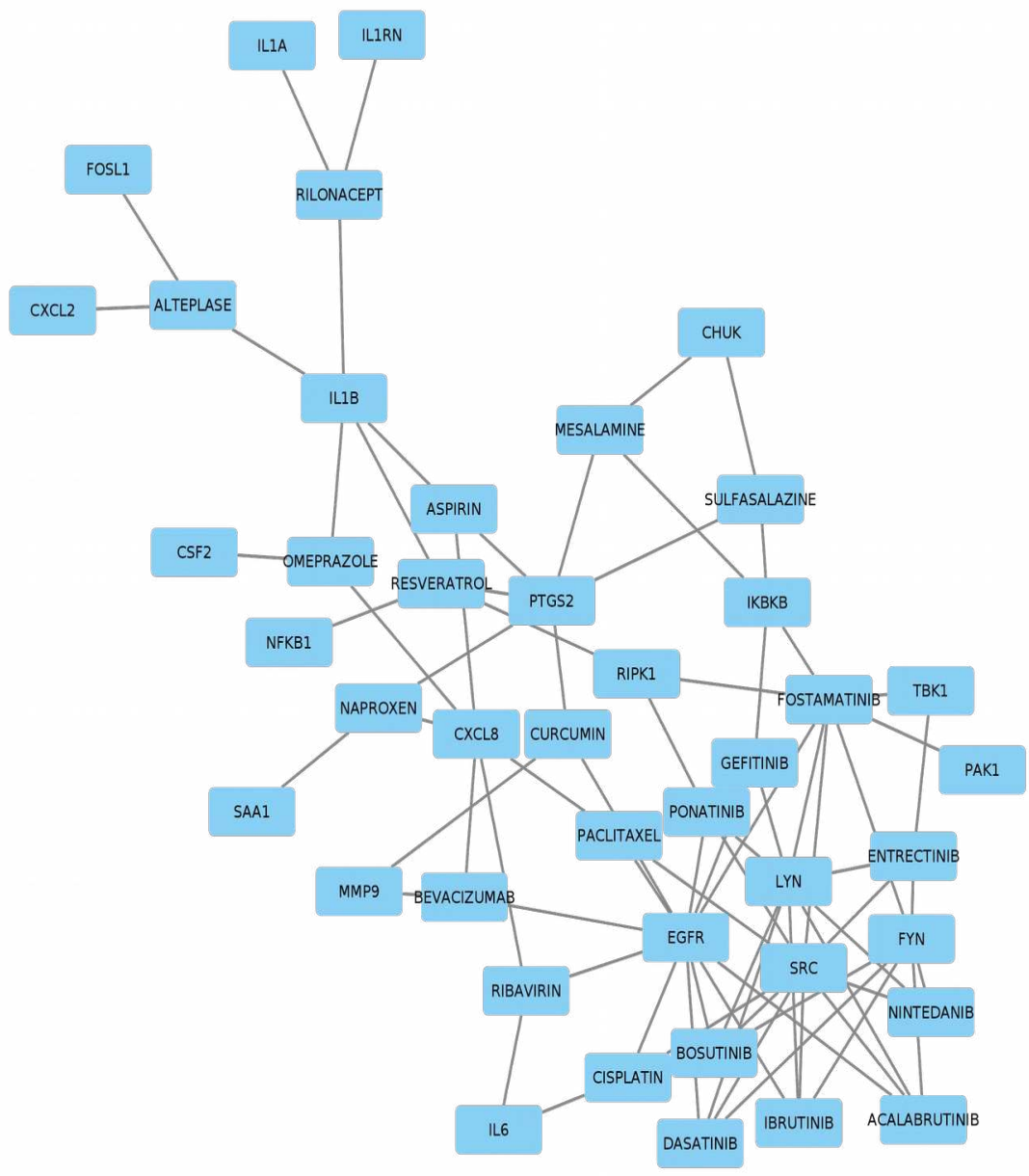

Figure 6. Drug-potential target network using Cytoscape.

Table 4. L1000FDW analysis

\begin{tabular}{|l|c|c|}
\hline Rank & Drugs & Similarity scores \\
\hline 1 & Sorafenib & -0.19496855345912 \\
\hline 2 & sirolimus & -0.19496855345912 \\
\hline 3 & Selumetinib & -0.182389937106918 \\
\hline 4 & fostamatinib & -0.176100628930818 \\
\hline 5 & dasatinib & -0.169811320754717 \\
\hline 6 & vemurafenib & -0.157232704402516 \\
\hline 7 & Neratinib & -0.150943396226415 \\
\hline 8 & Exemestane & -0.144654088050314 \\
\hline 9 & Bosutinib & -0.138364779874214 \\
\hline
\end{tabular}




\section{Functional Modules Analysis}

To understand the underlying pathologic pathways, we expanded the PPI network of DEGs to include 386 nodes, 5280 edges with an average node degree of 27.4 and avg. the local clustering coefficient of 0.587, and it's PPI enrichment p-value was < 1.0e-16 (Fig. S1). Then, the significant modules were identified via the MCODE using ClusterViz. The top eight functional clusters of modules were selected. KEGG pathway analysis and biological process enrichment analysis of each module was performed by STRING.

Module 1, with MCODE score = 32.789, consisted of 77 nodes and 1246 edges (Fig. S2).

The KEGG pathway analysis of this module indicated that IL-17 signaling pathway, axon guidance, TNF signaling pathway, cytokine-cytokine receptor interaction, NF-kappa B signaling pathway, and NOD-like receptor signaling pathway, are the most related pathways (Table S3). Biological process enrichment analysis identified that cell surface receptor signaling pathway, signal transduction, response to cytokine, ephrin receptor signaling pathway, and cytokine-mediated signaling pathway are the top enriched process for the first top module (Table S3).

Module 2, with MCODE score =16.049, consisted of 42 nodes and 329 edges (Fig. S3). The KEGG pathway analysis of this module indicated that NOD-like receptor signaling pathway, RIG-I-like receptor signaling pathway, NF-kappa B signaling pathway, TNF signaling pathway, influenza A, and hepatitis $\mathrm{C}$ are the most related pathways (Table S3). Biological process enrichment analysis identified that cytokine-mediated signaling pathway, response to cytokine, and cellular response to organic substance are the top enriched process for module 2 (Table S3).

Module 3, with MCODE score = 10.2, consisted of 11 nodes and 51 edges (Fig. S4). The KEGG pathway analysis of this module indicated that homologous recombination, non-homologous endjoining, and cellular senescence are the most related pathways (Table S3). Biological Process enrichment analysis identified that telomere capping, regulation of telomere maintenance, and negative 
regulation of telomere maintenance are the top enriched process for module 3 (Table S3).

Module 4, with MCODE score = 10, consisted of 10 nodes and 45 edges (Fig. S5). The KEGG pathway analysis of this module indicated that focal adhesion, regulation of actin cytoskeleton, leukocyte transendothelial migration, tight junction, vascular smooth muscle contraction, and platelet activation are the most related pathways (Table S3). Biological Process enrichment analysis identified that muscle contraction, and muscle organ development is the top enriched process for module 4 (Table S3).

Module 5, with MCODE score = 9.33, consisted of 10 nodes and 42 edges (Fig. S6). The KEGG pathway analysis of this module indicated that hepatitis C, NOD-like receptor signaling pathway, influenza A, herpes simplex infection, and measles are the most related pathways (Table S3).

Biological Process enrichment analysis identified that type I interferon signaling pathway, innate immune response, defense response to virus, and cellular response to cytokine stimulus are the top enriched process for module 5 (Table S3).

Module 6, with MCODE score = 9, consisted of 6 nodes and 36 edges (Fig. S7). For KEGG pathway enrichment analysis, this module wasn't enriched (Table S3). Biological Process enrichment analysis identified that peptide cross-linking and cornification were the top enriched process for module 6 (Table S3).

Module 7, with MCODE score $=7.778$, consisted of 10 nodes and 35 edges (Fig. S8). The KEGG pathway analysis of this module indicated that pathogenic Escherichia coli infection, gap junction, phagosome, apoptosis, and tight junction are the most related pathways (Table S3). Biological process enrichment analysis identified that microtubule-based process and cytoskeleton organization are the top enriched process for module 7 (Table S3).

Module 8, with MCODE score = 6.061, consisted of 10 nodes and 35 edges (Fig. S9). The KEGG pathway analysis of this module indicated that apoptosis, cytokine-cytokine receptor interaction, 
complement and coagulation cascades, natural killer cell-mediated cytotoxicity, and Jak-STAT signaling pathway are the most related pathways (Table S3). Biological process enrichment analysis identified that cell surface receptor signaling pathway, signal transduction and regulation of response to stress are the top enriched process for module 8 (Table S3).

\section{Discussion}

In this study, we used an integrated bioinformatics approach to analyse publicly available two gene expression profile of SARS-CoV-2 to discover potential novel insights regarding COVID-19 pathogenesis and therapeutics. We identified 187 overlapping DEGs for which KEGG pathway analysis and GO term analysis were performed and significant transcription factors and kinases were identified. From functional module analysis of the expanded PPI network of DEGs, many known pathogenic pathways and biological processes were identified as well as potential novel ones. Most of the DEGs in module 1 (NFKB2, CSF3, NFKB1, CSF2, TNFAIP3, CXCL1, CXCL2, MMP9, IL1RN, PTGS2, IL6, CCL20, SEMA7A, CXCL5, CXCL3, IL1A, ICAM1, IL1B) and module 2 (IL7R, EDN1, MAP3K8, PI3, BIRC3, IKBKE, CXCL16, BCL2A1, LIF, and CXCL6) were upregulated except two genes (IL6R and EPHA4) were down-regulated. Pathway analysis of DEGs, module 1 and module 2 revealed the significance of the following pathways, IL-17 signaling pathway, TNF signaling pathway, cytokine-cytokine receptor interaction, NF-kappa B signaling pathway, Rheumatoid arthritis, NODlike receptor signaling pathway, Toll-like receptor signaling pathway [2, 4, 25-36]. In addition to these pathways, axon guidance was highly enriched for module 1 . We found only one study which mentioned that axon guidance was top enriched for postmortem lung samples from patients who died of COVID-19 (7). In our finding, there were two DEGs involved in axon guidance (SEMA7A and EPHA4). SEMA7A was upregulated, however, EPHA4 was down-regulated. SEMA7A, a semorphin member, has been involved in both axon guidance [37] and regulation of immune cell activation [3839]. Sema7A and its receptor, PlexinC1 has been implicated in lung injury and fibrosis [40-42]. Roth 
JM et al (2016) found that proinflammatory cytokines and Sema7A can induce each other and explore the critical role of Sema7A in pulmonary inflammation [43]. Sema7A-plexinC1 signaling is considered a promising drug target for the treatment of acute lung injury [44]. With regards to EPHA4, there is a lack of studies that define the role of down-regulated EPHA4 in lung fibrosis and inflammation. Here, we considered that axon guidance, specially Sema7A and its receptor, plexinC1, is an attractive area for management COVID-19. From pathway analysis, it was found that COVID-19 share significant pathways with rheumatoid arthritis [45], Kaposi's sarcoma-associated herpesvirus infection, influenza A, hepatitis C, pathways in cancer [46-47], legionellosis, salmonella infection, herpes simplex infection, and measles. Apoptosis and RIG-I-like receptor signaling pathway were significantly enriched for module 2. This indicates that COVID 19 induces these important pathogenic pathways [48-52]. Apoptosis is a promising target for combating SARS-CoV-2 virus infection [48-51]. It was found that Telomere capping was the top enriched biological process enriched for module 3 (Table 5). TINF2 (TIN2) was up-regulated in our final DEGs. TIN2 is induced by NF-kB transcription factor [53] and is a core part of the Shelterin complex which protects telomeres and chromosomes. High TIN2 expression can produce telomere dysfunction-induced DNA damage and aberrant chromosomal alterations [54]. Telomere dysfunction limits the proliferative capacity of human cells by activation of DNA damage responses, inducing senescence or apoptosis [55]. Short telomere is manifested as pulmonary fibrosis-emphysema in adults [56]. Telomere dysfunction may provide a novel pathway for the pathogenesis of COVID-19. So, it's was found that homologous recombination, non-homologous end-joining, and cellular senescence are the only KEGG pathway enriched for module 3. Module 4 displays the interplay between platelets activation and leukocyte transendothelial migration which mediates thrombus formation a major contribution for the severity of COVID-19 [7, 57-58]. Focal adhesion, regulation of actin cytoskeleton, leukocyte transendothelial migration, tight junction, vascular smooth muscle contraction, and platelets activation are potential targets for COVID- 
19. MYLK was found to be downregulated and its role in the pathogenesis of COVID-2 is unclear. Besides, Genes of module 5 (MX2, IFI27, and GBP5) were upregulated and highlighted the role of type I interferon signaling pathway and innate immune response in COVID-19 pathogenesis [2, 5961]. SARS-CoV-2-infection up-regulated genes (TGM1, SPRR1B, LOR, SPRR2E, SPRR2D, SPRR1A, IVL, FLG, and SPRR2A) involved in cornification and peptide cross-linking which were enriched for module 6, this may lead to cell death and involved in lung fibrosis respectively [62-64]. Module 7 genes (TUBA1B, TUBB4B, TUBA1C, and TUBA1A) were up-regulated and involved in the microtubule-based process and cytoskeleton organization which may explore the potential significance of gap junction, phagosome, and tight junction in COVID-19 pathogenesis. Colchicine, which acts on tubulin, was repurposed for COVID-19 [65]. Genes of module 8 (SAA1, DDX58, SERPINB2, F3, TNFRSF10A, BID, PLAU, HBEGF, ANXA1, and PLAUR) were up-regulated, four of them (F3, PLAUR, PLAU, and SERPINB2) were involved in complement and coagulation cascades and considered of potential importance in coagulation disorders associated with COVID-19 infection. Many papers explored the significance of Jak-STAT signaling pathway, and natural killer cell-mediated cytotoxicity [66-67].

From the unexpanded PPI network of DEGs, 18 hub genes (IL6, CXCL8, CXCL1, CCL20, CXCL2, CXCL3, IL1B, IL1A, MMP9, CXCL5, CSF3, ICAM1, IL1RN, CSF2, PTGS2, SAA1, NFKB1, and TNFAIP3) were identified by the overlap of three methods and it was found that these hub genes were highly interactive with each other. Hub genes along with the top enriched transcription factors and kinases for DEGs were used for constructing drug-potential target network. Drugs are ranked according to the degree. Drugs with a high degree $(>=3)$ were prioritized. The top potential drugs as Fostamatinib (Degree $=8)$, Acalabrutinib (Degree $=4$ ), and Ibrutinib (Degree $=4$ ) were already suggested to be repurposed for COVID-19 in recent papers [68-70]. Phase II clinical trials were conducted to study the safety and efficacy of Fostamatinib, Acalabrutinib, Ibrutinib, and 
Bevacizumab with (degree $=3$ ) for hospitalized patients with COVID-19 (ClinicalTrials.gov). Another top potential drug, Nintedanib with (degree $=3$ ), is under phase III for treatment of SARS-Cov-2 Induced Pulmonary Fibrosis (ClinicalTrials.gov). Sulfasalazine was considered a potential drug [71]. Other top potential drugs as Dasatinib, Bosutinib, Entrectinib, Ponatinib, Vandetanib, Gefitinib, Mesalamine, Rilonacept, Alteplase, Omeprazole, Naproxen, Cisplatin, and Ribavirin are considered novel potential drugs for COVID-19. There is a controversy about using Ribavirin in COVID-19 (7275). Some papers addressed the beneficial effects of the two botanical-derived drugs, Resveratrol and Curcumin, in COVID-19 [76-79].Along with the degree, we added another dimension for prioritization. We selected those novel potential drugs which act on the same targets as those under clinical investigation. It was found that Dasatinib and Bosutinib shared the same targets (EGFR-SRCFYN-LYN) as with Acalabrutinib and Ibrutinib (EGFR-SRC-FYN-LYN) whereas targets of Entrectinib (SRC-TBK1-FYN-LYN), Ponatinib (EGFR-RIPK1-SRC-LYN), and Vandetanib (EGFRSRC-FYN) included three of them (Table 3). L1000FDW analysis added new potential drugs as in table 5. Fostamatinib, Dasatinib, and Bosutinib were common between the two methods (drugpotential target network and L1000FDW analysis). Other potential drugs revealed by L1000FDW analysis were Sorafenib, Sirolimus [80], Selumetinib [80], Neratinib [81], Vemurafenib, Exemestane, and Bosutinib.

\section{Conclusion}

In this study, we tried to decipher potentially hidden parts of the pathogenesis of COVID-19 as well as repurposing FDA-approved drugs using an integrative bioinformatics approach. Besides the wellknown pathogenic pathways like IL-17 signaling pathway, TNF signaling pathway, Cytokine-cytokine receptor interaction, NF-kappa B signaling pathway, NOD-like receptor signaling pathway, Toll-like receptor signaling pathway, platelets activation and leukocyte transendothelial migration, axon guidance is a potential pathogenic pathway. Sema7A and its receptor, plexinC1, is a potential novel 
drug target. Sema7A may exacerbate cytokines production. Another potential novel pathway is related to TINF2 overexpression which may induce potential telomere dysfunction and hence DNA damage that may exacerbate lung fibrosis. We identified repurposed drugs that are under investigation for hospitalized patients with COVID-19 and new potential drugs such as Dasatinib, Bosutinib, Entrectinib, Ponatinib, and Vandetanib were prioritized based on two factors (The degree and the similarity of targets with those drugs which were under clinical investigation). Others like Sorafenib, Vemurafenib, and Exemestane were identified as potential novel drugs using L1000FDW analysis. Our findings provide new insight into the mechanisms of COVID-19 complications as well as potential new drugs. Further validation of these findings is needed. Although we didn't do an experimental validation, finding known pathogenic pathways and drugs currently in clinical trials for COVID-19 in our analysis is one way of validation for our method.

\section{Supplementary Materials}

Fig. S1: Expanded PPI network of DEGs, Fig. S2: Module 1, Fig. S3: Module 2, Fig. S4: Module 3, Fig. S5: Module 4, Fig. S6: Module 5, Fig. S7: Module 6, Fig. S8: Module 7, Fig. S9: Module 8, Table S1. List of differentially expressed genes (DEGs), Table S2. List of Drugs ranked by degree, Table S3. KEGG pathway, GO Biological Process of functional modules, and DEGs involved in each module.

\section{Funding}

This research was funded by Deanship of Scientific Research, Imam Abdulrahman Bin Faisal University, Dammam, Saudi Arabia, Grant No. Covid19-2020-001-BASRC”.

\section{Acknowledgements}

We are grateful for the GEO, KEGG, STRING, L1000FDW, and DGIdb databases. Also, we wish to thank Dr Ahmed Mostafa (National Research Center, Egypt) for his support and suggestion.

\section{Author Contributions}


Conceptualization, A.M., S.E., and M.E.; methodology, S.E. .; formal analysis, S.E.; writing—original draft preparation, S.E. .; writing-review and editing, A.M., S.E., and M.E ; visualization, S.E., and M.E. All authors have read and agreed to the published version of the manuscript.

\section{Institutional Review Board Statement}

Not applicable.

\section{Informed Consent Statement}

Not applicable.

\section{Data Availability Statement}

\section{Not applicable.}

\section{Conflicts of Interest}

The authors declare no conflict of interest.

\section{Reference}

1. C. Huang, Y. Wang, X. Li, L. Ren, J. Zhao, Y. Hu, L. Zhang, G. Fan, J. Xu, X. Gu, Z. Cheng, T. Yu, J. Xia, Y. Wei, W. Wu, X. Xie, W. Yin, H. Li, M. Liu, Y. Xiao, H. Gao, L. Guo, J. Xie, G. Wang, R. Jiang, Z. Gao, Q. Jin, J. Wang, B. Cao, Clinical features of patients infected with 2019 novel coronavirus in Wuhan, China, Lancet 395 (10223) (2020) 497-506.

2. D. Blanco-Melo, B.E. Nilsson-Payant, W.C. Liu, et al., Imbalanced host response to SARS-CoV-2 drives development of COVID-19, Cell 181 (5) (2020) 1036-1045.e9, https://doi.org/10.1016/j. Cell.2020.04.026.

3. Vanderheiden A, Ralfs P, Chirkova T, Upadhyay AA, Zimmerman MG, Bedoya S, Aoued H, Tharp GM, Pellegrini KL, Manfredi C, Sorscher E, Mainou B, Lobby JL, Kohlmeier JE, Lowen AC, Shi P-Y, Menachery VD, Anderson LJ, Grakoui A, Bosinger SE, Suthar MS. 2020. Type I and type III interferons restrict SARS-CoV-2 infection of human airway epithelial cultures. J Virol 94:e00985-20. https://doi.org/10.1128/JVI.00985-20 
4. Peipei Song, Wei Li, Jianqin Xie, Yanlong Hou, Chongge You. Cytokine storm induced by SARSCoV-2. Clinica Chimica Acta 509 (2020) 280-287.

5. Z. Zhou, L. Ren, L. Zhang, J. Zhong, Y. Xiao, Z. Jia, L. Guo, J. Yang, C. Wang, S. Jiang, D. Yang, G. Zhang, H. Li, F. Chen, Y. Xu, M. Chen, Z. Gao, J. Dong, B. Liu, X. Zhang, W. Wang, K. He, Q. Jin, M. Li, J. Wang, Z. Zhou, L. Ren, L. Zhang, J. Zhong, Y. Xiao, Z. Jia, L. Guo, J. Yang, C. Wang, S. Jiang, D. Yang, G. Zhang, H. Li, F. Chen, Y. Xu, M. Chen, Z. Gao, J. Dong, B. Liu, X. Zhang, W. Wang, K. He, Q. Jin, M. Li, J. Wang, Heightened innate immune responses in the respiratory tract of COVID-19 patients, Cell Host Microbe 27 (6) (2020) 883-890.

6. R.M. Golonka, P. Saha, B.S. Yeoh, S. Chattopadhyay, A.T. Gewirtz, B. Joe, M. VijayKumar, Harnessing innate immunity to eliminate SARS-CoV-2 and ameliorate COVID-19 disease, Physiol. Genomics 52 (5) (2020) 217-221.

Y. Shi, Y. Wang, C. Shao, J. Huang, J. Gan, X. Huang, E. Bucci, M. Pi

7. Wu M, Chen Y, Xia H, Wang C, Tan CY, Cai X, Liu Y, Ji F, Xiong P, Liu R, Guan Y, Duan Y, Kuang D, Xu S, Cai H, Xia Q, Yang D, Wang MW, Chiu IM, Cheng C, Ahern PP, Liu L, Wang G, Surana NK, Xia T, Kasper DL. Transcriptional and proteomic insights into the host response in fatal COVID-19 cases. Proc Natl Acad Sci U S A. 2020 Nov 10;117(45):28336-28343. doi: 10.1073/pnas.2018030117. Epub 2020 Oct 20. PMID: 33082228; PMCID: PMC7668053.

8. Wang, Dengchuan BSc, MSc; Liu, Shengshuo BSc; Xu, Shi PhD. Identification of hub genes, key pathways, and therapeutic agents in Hutchinson-Gilford Progeria syndrome using bioinformatics analysis, Medicine: February 2020 - Volume 99 - Issue 7 - p e19022 doi:

10.1097/MD.0000000000019022.

9. Yang D, He Y, Wu B, et al. Integrated bioinformatics analysis for the screening of hub genes and therapeutic drugs in ovarian cancer. J Ovarian Res. 2020;13(1):10. doi: 10.1186/s13048-020-0613- 
10. El-aarag, S.A., Mahmoud, A., Hashem, M.H. et al. In silico identification of potential key regulatory factors in smoking-induced lung cancer. BMC Med Genomics 10, 40 (2017). https://doi.org/ 10.1186/s12920-017-0284-Z.

11. Andrews, S. (n.d.). FastQC A Quality Control tool for High Throughput Sequence Data. Retrieved from http://www.bioinformatics.babraham.ac.uk/projects/fastqc.

12. Bolger, A. M., Lohse, M., \& Usadel, B. (2014). Trimmomatic: a flexible trimmer for Illumina sequence data. Bioinformatics, 30(15), 2114â€“2120. Retrieved from http://dx.doi.org/10.1093/bioinformatics/btu170.

13. Kim, D., Langmead, B., \& Salzberg, S. L. (2015). HISAT: a fast spliced aligner with low memory requirements. Nature Methods, 12(4), 357â€“360. Retrieved from http://dx.doi.org/10.1038/nmeth.331.

14. Institute, B. (n.d.). Picard. Broad Institute, GitHub repository. GitHub. Retrieved from http://broadinstitute.github.io/picard/Institute, B. (n.d.). Picard. Broad Institute, GitHub repository. GitHub. Retrieved from http://broadinstitute.github.io/picard/.

15. Anders, S., Pyl, P. T., \& Huber, W. (2014). HTSeq-a Python framework to work with highthroughput sequencing data. Bioinformatics, 31(2), 166â€“169. Retrieved from http://dx.doi.org/10.1093/bioinformatics/btu638.

16. Love, M. I., Huber, W., \& Anders, S. (2014). Moderated estimation of fold change and dispersion for RNA-seq data with DESeq2. Genome Biology, 15(12). Retrieved from http://dx.doi.org/10.1186/s13059-014-0550-8.

17. Keenan AB, Torre D, Lachmann A, Leong AK, Wojciechowicz M, Utti V, Jagodnik K, Kropiwnicki E, Wang Z, Ma'ayan A (2019) ChEA3: transcription factor enrichment analysis by orthogonal omics integration. Nucleic Acids Research. doi: $\underline{10.1093 / \text { nar/gkz446. }}$. 
18. Shannon P, Markiel A, Ozier O, Baliga NS, Wang JT, Ramage D, Amin N, Schwikowski B, Ideker T. Cytoscape: a software environment for integrated models of biomolecular interaction networks Genome Research 2003 Nov; 13(11):2498-504.

19. Tang Y, Li M, Wang J, Pan Y and Wu FX: CytoNCA: A Cytoscape plugin for centrality analysis and evaluation of protein interaction networks. Biosystems. 127:67-72. 2015. View Article: Google Scholar: $\underline{\text { PubMed/NCBI }}$

20. Chin $\mathrm{CH}$, Chen SH, Wu HH, Ho CW, Ko MT, Lin CY. cytoHubba: identifying hub objects and sub-networks from complex interactome. BMC Syst Biol. 2014;8 Suppl 4(Suppl 4): S11. doi:

10.1186/1752-0509-8-S4-S11. Epub 2014 Dec 8. PMID: 25521941; PMCID: PMC4290687.

21. Wang Z, Lachmann A, Keenan AB, Ma’Ayan A. L1000FWD: Fireworks visualization of druginduced transcriptomic signatures. Bioinformatics 2018;34:2150-2.

doi:10.1093/bioinformatics/bty060.

22. Bader, G.D., Hogue, C.W. An automated method for finding molecular complexes in large protein interaction networks. BMC Bioinformatics 4, 2 (2003). https://doi.org/10.1186/1471-2105-4-2.

23. Wang J, Zhong J, Chen G, Li M, Wu FX, Pan Y. ClusterViz: A Cytoscape APP for Cluster Analysis of Biological Network. IEEE/ACM Trans Comput Biol Bioinform. 2015 Jul-Aug;12(4):815-22. doi: 10.1109/TCBB.2014.2361348. PMID: 26357321.

24. Oliveros, J.C. (2007-2015) Venny. An interactive tool for comparing lists with Venn's diagrams. https://bioinfogp.cnb.csic.es/tools/venny/index.html

25. Appelberg S, Gupta S, Svensson Akusjärvi S, Ambikan AT, Mikaeloff F, Saccon E, Végvári Á, Benfeitas R, Sperk M, Ståhlberg M, Krishnan S, Singh K, Penninger JM, Mirazimi A, Neogi U. Dysregulation in Akt/mTOR/HIF-1 signaling identified by proteo-transcriptomics of SARS-CoV-2 infected cells. Emerg Microbes Infect. 2020 Dec;9(1):1748-1760. doi:

10.1080/22221751.2020.1799723. PMID: 32691695; PMCID: PMC7473213. 
26. Hemmat N, Asadzadeh Z, Ahangar NK, Alemohammad H, Najafzadeh B, Derakhshani A, Baghbanzadeh A, Baghi HB, Javadrashid D, Najafi S, Ar Gouilh M, Baradaran B. The roles of signaling pathways in SARS-CoV-2 infection; lessons learned from SARS-CoV and MERS-CoV. Arch Virol. 2021 Jan 18:1-22. doi: 10.1007/s00705-021-04958-7. Epub ahead of print. PMID: 33462671; PMCID: PMC7812983.

27. Hojyo, S., Uchida, M., Tanaka, K. et al. How COVID-19 induces cytokine storm with high mortality. Inflamm Regener 40, 37 (2020). https://doi.org/10.1186/s41232-020-00146-3

28. Hariharan A, Hakeem AR, Radhakrishnan S, Reddy MS, Rela M. The Role and Therapeutic Potential of NF-kappa-B Pathway in Severe COVID-19 Patients. Inflammopharmacology. 2020 Nov 7:1-10. doi: 10.1007/s10787-020-00773-9. Epub ahead of print. PMID: 33159646; PMCID: PMC7648206.

29. Chen I-Y, Moriyama M, Chang M-F, Ichinohe T. Severe acute respiratory syndrome coronavirus viroporin 3a activates the NLRP3 inflammasome. Front Microbiol. (2019) 10:50. doi:

10.3389/fmicb.2019.00050

30. Tong Y, Ding Z-H, Zhan F-X, Cai L, Yin X, Ling J-L, et al. The NLRP3 inflammasome and stroke. Int J Clin Exp Med. (2015) 8:4787-94.

31. Yang Y, Wang H, Kouadir M, Song H, Shi F. Recent advances in the mechanisms of NLRP3 inflammasome activation and its inhibitors. Cell Death Dis. (2019) 10:128. doi: 10.1038/s41419-019$1413-8$

32. Shah A. Novel coronavirus-induced NLRP3 inflammasome activation: a potential drug target in the treatment of COVID-19. Front Immunol. (2020) 11:1021. doi: 10.3389/fimmu.2020.01021

33. Freeman TL, Swartz TH. Targeting the NLRP3 inflammasome in severe COVID-19. Front Immunol. (2020) 11:1518. doi: 10.3389/fimmu.2020.01518 
34. Brandão SCS, Ramos JOX, Dompieri LT, Godoi ETAM, Figueiredo JL, Sarinho ESC,

Chelvanambi S, Aikawa M. Is Toll-like receptor 4 involved in the severity of COVID-19 pathology in patients with cardiometabolic comorbidities? Cytokine Growth Factor Rev. 2020 Sep 21:S13596101(20)30205-7. doi: 10.1016/j.cytogfr.2020.09.002. Epub ahead of print. PMID: 32988728; PMCID: PMC7505161.

35. Shibabaw T. Inflammatory Cytokine: IL-17A Signaling Pathway in Patients Present with COVID19 and Current Treatment Strategy. Journal of Inflammation Research. 2020 ;13:673-680. DOI: 10.2147/jir.s278335.

36. Maxwell AJ, Ding J, You Y, Dong Z, Chehade H, Alvero A, Mor Y, Draghici S, Mor G. Identification of key signaling pathways induced by SARS-CoV2 that underlie thrombosis and vascular injury in COVID-19 patients. J Leukoc Biol. 2020 Nov 26:10.1002/JLB.4COVR0920552RR. doi: 10.1002/JLB.4COVR0920-552RR. Epub ahead of print. PMID: 33242368; PMCID: PMC7753679.

37. Pasterkamp RJ, Peschon JJ, Spriggs MK and Kolodkin AL, Semaphorin 7A promotes axon outgrowth through integrins and MAPKs. Nature 2003. 424: 398-405.

38. Suzuki K, Okuno T, Yamamoto M, Pasterkamp RJ, Takegahara N, Takamatsu H, Kitao T et al., Semaphorin 7A initiates T-cell-mediated inflammatory responses through alpha1beta1 integrin. Nature 2007. 446: 680-684.

39. Czopik AK, Bynoe MS, Palm N, Raine CS and Medzhitov R, Semaphorin 7A is a negative regulator of T cell responses. Immunity 2006. 24: 591-600.

40. Morote-Garcia JC, Napiwotzky D, Köhler D, Rosenberger P. Endothelial Semaphorin 7A promotes neutrophil migration during hypoxia. Proc Natl Acad Sci USA 2012;109:14146-14151.

41. Granja T, Köhler D, Mirakaj V, Nelson E, König K, Rosenberger P. Crucial role of Plexin C1 for 
pulmonary inflammation and survival during lung injury. Mucosal Immunol 2014;7:879-891.

42. Peng X, Moore M, Mathur A, Zhou Y, Sun H, Gan Y, et al. Plexin C1 deficiency permits synaptotagmin 7-mediated macrophage migration and enhances mammalian lung fibrosis.

43. Roth JM, Köhler D, Schneider M, Granja TF, Rosenberger P. Semaphorin 7A aggravates pulmonary inflammation during lung injury. PLoS One 2016;11:e0146930.

44. H. Movassagh, F. Khadem, A. S. Gounni, Semaphorins and their roles in airway biology: Potential as therapeutic targets. Am. J. Respir. Cell Mol. Biol. 58, 21-27 (2018).

45. Favalli EG, Ingegnoli F, De Lucia O, Cincinelli G, Cimaz R, Caporali R. COVID-19 infection and rheumatoid arthritis: Faraway, so close! Autoimmun Rev. 2020 May;19(5):102523. doi:

10.1016/j.autrev.2020.102523. Epub 2020 Mar 20. PMID: 32205186; PMCID: PMC7102591

46. Chen J, Dai L, Barrett L, Post SR, Qin Z. SARS-CoV-2 proteins and anti-COVID-19 drugs induce lytic reactivation of an oncogenic virus. bioRxiv [Preprint]. 2020 Oct 2:2020.10.02.324228. doi:

10.1101/2020.10.02.324228. PMID: 33024968; PMCID: PMC7536871.

47. Saha A, Anirvan P. Cancer progression in COVID-19: integrating the roles of renin angiotensin aldosterone system, angiopoietin-2, heat shock protein-27 and epithelial mesenchymal transition. Ecancermedicalscience. 2020 Sep 9;14:1099. doi: 10.3332/ecancer.2020.1099. PMID: 33082849; PMCID: PMC7532023.

48. Donia, A., Bokhari, H. Apoptosis induced by SARS-CoV-2: can we target it?. Apoptosis (2021). https://doi.org/10.1007/s10495-021-01656-2.

49. Ren Y et al (2020) The ORF3a protein of SARS-CoV-2 induces apoptosis in cells. Cell Mol Immunol 17:881-883.

50. Taghiloo S, et al. (2020) Apoptosis and immunophenotyping of peripheral blood lymphocytes in Iranian COVID 19 patients: clinical and laboratory characteristics. J Med Virol.

51. Li S et al (2020) SARS-CoV-2 triggers inflammatory responses and cell death through caspase-8 
activation. Signal Transd Targeted Ther 5:1-10.

52. Onomoto, K., Onoguchi, K. \& Yoneyama, M. Regulation of RIG-I-like receptor-mediated signaling: interaction between host and viral factors. Cell Mol Immunol (2021).

https://doi.org/10.1038/s41423-020-00602-7Onomoto, K., Onoguchi, K. \& Yoneyama, M. Regulation of RIG-I-like receptor-mediated signaling: interaction between host and viral factors. Cell Mol Immunol (2021). https://doi.org/10.1038/s41423-020-00602-7

53. Xin Z-T, Carroll KA, Kumar N, Song K, Ly H (2011) Transcriptional Activation of TINF2, a Gene Encoding the Telomere-Associated Protein TIN2, by Sp1 and NF-kB Factors. PLoS ONE 6(6): e21333. doi:10.1371/journal.pone.0021333

54. Fu, F., Hu, H., Yang, S., \& Liang, X. (2018). Effects of TIN2 on telomeres and chromosomes in the human gastric epithelial cell line GES-1. Oncology Letters, 15, 5161-5166. https://doi.org/10.3892/ol.2018.7927

55. Jiang H, Schiffer E, Song Z, Wang J, Zürbig P, Thedieck K, Moes S, Bantel H, Saal N, Jantos J, Brecht M, Jenö P, Hall MN, Hager K, Manns MP, Hecker H, Ganser A, Döhner K, Bartke A, Meissner C, Mischak H, Ju Z, Rudolph KL. Proteins induced by telomere dysfunction and DNA damage represent biomarkers of human aging and disease. Proc Natl Acad Sci U S A. 2008 Aug 12;105(32):11299-304. doi: 10.1073/pnas.0801457105. Epub 2008 Aug 11. Erratum in: Proc Natl Acad Sci U S A. 2018 Apr 2;: PMID: 18695223; PMCID: PMC2516278.

56. Trăilă D, Mlădinescu OF, Oancea C, Tudorache V. Short telomeres in pulmonary fibrosis: from genetics to clinical significance. Pneumologia. 2015 Jan-Mar;64(1):8, 11-3. PMID: 26016050 57. Tasnimul Alam Taz, Kawsar Ahmed, Bikash Kumar Paul, Md Kawsar, Nargis Aktar, S M Hasan Mahmud, Mohammad Ali Moni, Network-based identification genetic effect of SARS-CoV-2 infections to Idiopathic pulmonary fibrosis (IPF) patients, Briefings in Bioinformatics, 2020;, bbaa235, https://doi.org/10.1093/bib/bbaa235 
58. Coenen DM, Mastenbroek TG, Cosemans JMEM. Platelet interaction with activated endothelium: mechanistic insights from microfluidics. Blood. 2017 Dec 28;130(26):2819-2828. doi: 10.1182/blood2017-04-780825. Epub 2017 Oct 10. PMID: 29018081

59. Sa Ribero M, Jouvenet N, Dreux M, Nisole S (2020) Interplay between SARS-CoV-2 and the type I interferon response. PLoS Pathog 16(7): e1008737. https://doi.org/10.1371/journal.ppat.1008737 60. Bourdon, M., Manet, C. \& Montagutelli, X. Host genetic susceptibility to viral infections: the role of type I interferon induction. Genes Immun 21, 365-379 (2020). https://doi.org/10.1038/s41435-020$\underline{00116-2}$

61. Lei X, Dong X, Ma R, Wang W, Xiao X, Tian Z, Wang C, Wang Y, Li L, Ren L, Guo F, Zhao Z, Zhou Z, Xiang Z, Wang J. Activation and evasion of type I interferon responses by SARS-CoV-2. Nat Commun. 2020 Jul 30;11(1):3810. doi: 10.1038/s41467-020-17665-9. PMID: 32733001; PMCID: PMC7392898.

62. Kang K, Kim HH, Choi Y. Tiotropium is Predicted to be a Promising Drug for COVID-19 Through Transcriptome-Based Comprehensive Molecular Pathway Analysis. Viruses. 2020 Jul 20;12(7):776. doi: 10.3390/v12070776. PMID: 32698440; PMCID: PMC7412475.

63. Eckhart L., Lippens S., Tschachler E., Declercq W. Cell death by cornification. Biochim. Biophys. Acta (BBA)—Bioenerg. 2013;1833:3471-3480. doi: 10.1016/j.bbamcr.2013.06.010.

64. Lorand L., Graham R.M. Transglutaminases: Crosslinking enzymes with pleiotropic functions. Nat. Rev. Mol. Cell Boil. 2003;4:140-156. doi: 10.1038/nrm1014.

65. Schlesinger N, Firestein BL, Brunetti L. Colchicine in COVID-19: an Old Drug, New Use. Curr Pharmacol Rep. 2020 Jul 18:1-9. doi: 10.1007/s40495-020-00225-6. Epub ahead of print. PMID: 32837853; PMCID: PMC7367785.

66. Luo W, Li YX, Jiang LJ, Chen Q, Wang T, Ye DW. Targeting JAK-STAT Signaling to Control Cytokine Release Syndrome in COVID-19. Trends Pharmacol Sci. 2020 Aug;41(8):531-543. doi: 
10.1016/j.tips.2020.06.007. Epub 2020 Jun 17. PMID: 32580895; PMCID: PMC7298494.

67. van Eeden C, Khan L, Osman MS, Cohen Tervaert JW. Natural Killer Cell Dysfunction and Its Role in COVID-19. Int J Mol Sci. 2020 Sep 1;21(17):6351. doi: 10.3390/ijms21176351. PMID: 32883007; PMCID: PMC7503862.

68. Neha Tabassum, Hua Zhang, Justin Stebbing. Repurposing Fostamatinib to Combat SARS-CoV-2Induced Acute Lung Injury, Cell Reports Medicine, Volume 1, Issue 8, 2020, 100145, ISSN 2666-3791, https://doi.org/10.1016/j.xcrm.2020.100145.

69. Treon SP, Castillo JJ, Skarbnik AP, Soumerai JD, Ghobrial IM, Guerrera ML, Meid K, Yang G. The BTK inhibitor ibrutinib may protect against pulmonary injury in COVID-19-infected patients. Blood. 2020 May 21;135(21):1912-1915. doi: 10.1182/blood.2020006288. PMID: 32302379; PMCID: PMC7243149

70. Roschewski M, Lionakis MS, Sharman JP, Roswarski J, Goy A, Monticelli MA, Roshon M, Wrzesinski SH, Desai JV, Zarakas MA, Collen J, Rose K, Hamdy A, Izumi R, Wright GW, Chung KK, Baselga J, Staudt LM, Wilson WH. Inhibition of Bruton tyrosine kinase in patients with severe COVID-19. Sci Immunol. 2020 Jun 5;5(48):eabd0110. doi: 10.1126/sciimmunol.abd0110. Epub 2020 Jun 5. PMID: 32503877; PMCID: PMC7274761.

71. Loganathan T, Ramachandran S, Shankaran P, Nagarajan D, Mohan S S. 2020. Host transcriptomeguided drug repurposing for COVID-19 treatment: a meta-analysis based approach. PeerJ 8:e9357 https://doi.org/10.7717/peerj.9357

72. Tong S, Su Y, Yu Y, Wu C, Chen J, Wang S, Jiang J. Ribavirin therapy for severe COVID-19: a retrospective cohort study. Int J Antimicrob Agents. 2020 Sep;56(3):106114. doi:

10.1016/j.ijantimicag.2020.106114. Epub 2020 Jul 23. PMID: 32712334; PMCID: PMC7377772. 73. Al-Tawfiq JA, Momattin H, Dib J, Memish ZA. Ribavirin and interferon therapy in patients infected with the Middle East respiratory syndrome coronavirus: an observational study. Int J Infect 
Dis. 2014 Mar;20:42-6. doi: 10.1016/j.ijid.2013.12.003. Epub 2014 Jan 6. PMID: 24406736; PMCID: PMC7110882.

74. Altay Unal M et al . 2020. Ribavirin shows antiviral activity against SARS-CoV-2 and downregulates the activity of TMPRSS2 and the expression of ACE2 In Vitro. bioRxiv 2020.12.04.410092; doi: https://doi.org/10.1101/2020.12.04.410092

75. Hung IF, Lung KC, Tso EY, Liu R, Chung TW, Chu MY, Ng YY, Lo J, Chan J, Tam AR, Shum HP, Chan V, Wu AK, Sin KM, Leung WS, Law WL, Lung DC, Sin S, Yeung P, Yip CC, Zhang RR, Fung AY, Yan EY, Leung KH, Ip JD, Chu AW, Chan WM, Ng AC, Lee R, Fung K, Yeung A, Wu TC, Chan JW, Yan WW, Chan WM, Chan JF, Lie AK, Tsang OT, Cheng VC, Que TL, Lau CS, Chan KH, To KK, Yuen KY. Triple combination of interferon beta-1b, lopinavir-ritonavir, and ribavirin in the treatment of patients admitted to hospital with COVID-19: an open-label, randomised, phase 2 trial. Lancet. 2020 May 30;395(10238):1695-1704. doi: 10.1016/S0140-6736(20)31042-4. Epub 2020 May 10. PMID: 32401715; PMCID: PMC7211500.

76. Manoharan Y., Haridas V., Vasanthakumar K., Muthu S., Thavoorullah F.F., Shetty P. Curcumin: A wonder drug as a preventive measure for covid19 management. Indian Journal of Clinical.

77. Zahedipour F, Hosseini SA, Sathyapalan T, Majeed M, Jamialahmadi T, Al-Rasadi K, Banach M, Sahebkar A. Potential effects of curcumin in the treatment of COVID-19 infection. Phytother Res. 2020 Nov;34(11):2911-2920. doi: 10.1002/ptr.6738. Epub 2020 Jun 23. PMID: 32430996; PMCID: PMC7276879.

78. Ramdani LH, Bachari K. Potential therapeutic effects of Resveratrol against SARS-CoV-2. Acta Virol. 2020;64(3):276-280. doi: 10.4149/av_2020_309. PMID: 32985211.

79. Hoang T. An approach of fatty acids and resveratrol in the prevention of COVID-19 severity. Phytother Res. 2020 Nov 17:10.1002/ptr.6956. doi: 10.1002/ptr.6956. Epub ahead of print. PMID: 33200839; PMCID: PMC7753401. 
80. Fagone P, Ciurleo R, Lombardo SD, Iacobello C, Palermo CI, Shoenfeld Y, Bendtzen K, Bramanti P, Nicoletti F. Transcriptional landscape of SARS-CoV-2 infection dismantles pathogenic pathways activated by the virus, proposes unique sex-specific differences and predicts tailored therapeutic strategies. Autoimmun Rev. 2020 Jul;19(7):102571. doi: 10.1016/j.autrev.2020.102571. Epub 2020 May 3. PMID: 32376402; PMCID: PMC7252184.

81. Victor S.Stroylov and Igor V.Svitanko. Computational identification of disulfiram and neratinib as putative SARS-CoV-2 main protease inhibitors. Mendeleev Communications, Volume 30, Issue 4, July-August 2020, Pages 419-420 


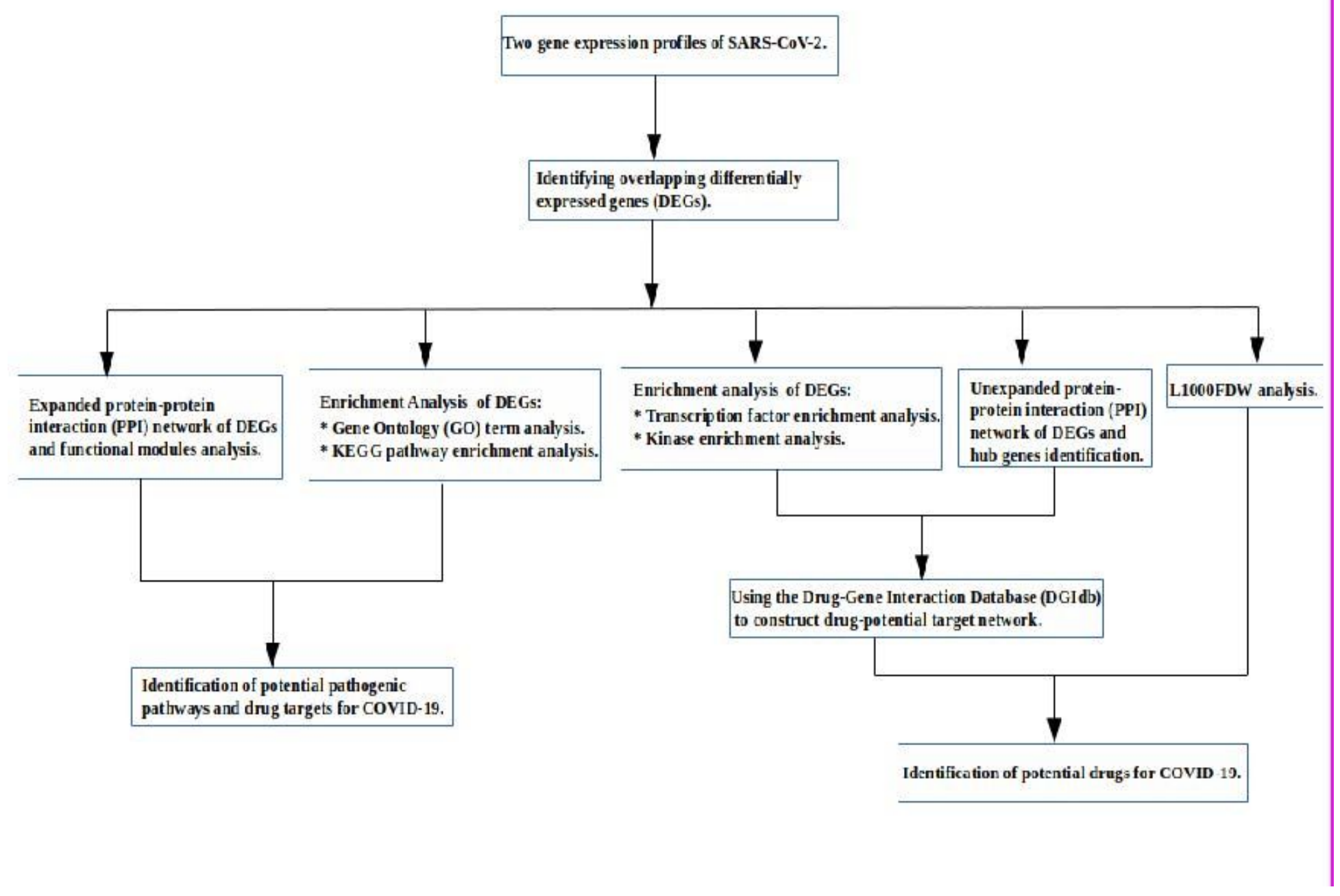

\section{Figure 1}

we present a Flow chart of our integrated novel bioinformatics approach to identify potential pathogenic pathways, drug targets, and drug candidates for COVID-19. 

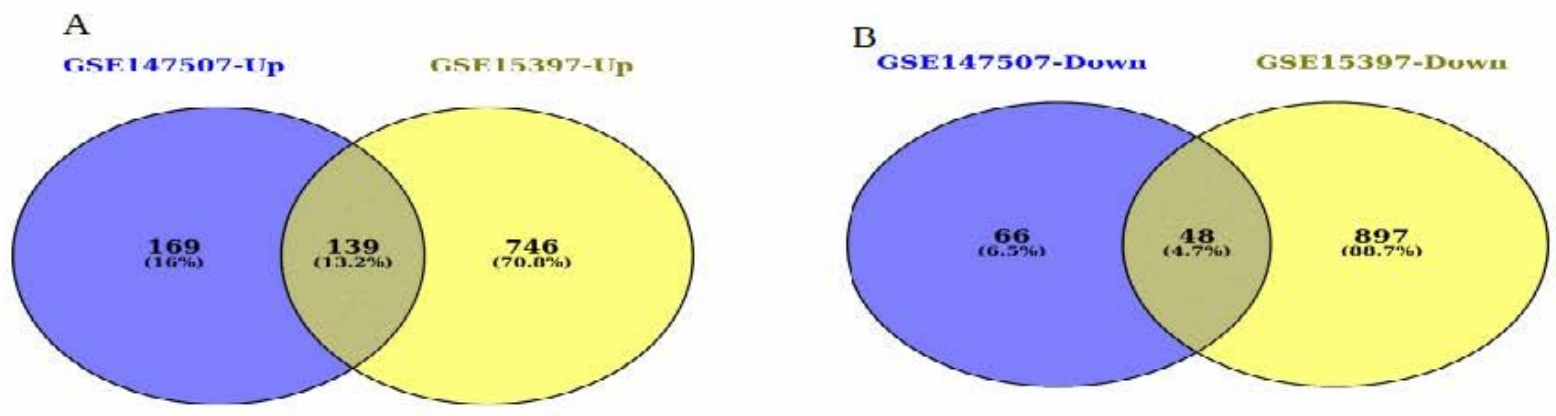

\section{Figure 2}

Venn diagram for overlapping differentially expressed genes (DEGs) based on two datasets. (A) the intersection of upregulated DEGs and (B) the intersection of downregulated DEGs identified from the two datasets, namely, GSE147507 and GSE15397.

A

IL-17 signaling pathway
TNF signaling pathway
Cytokine-cytokine receptor interactio
NF-kappa B signaling pathway
Rheumatoid arthrits
NOD-ilike receptor signaling pathway
Legionellosi

Transcriptional misregulation in cancer Salmonella infection

Kaposi's sarcoma-associated herpesvirus infection

C
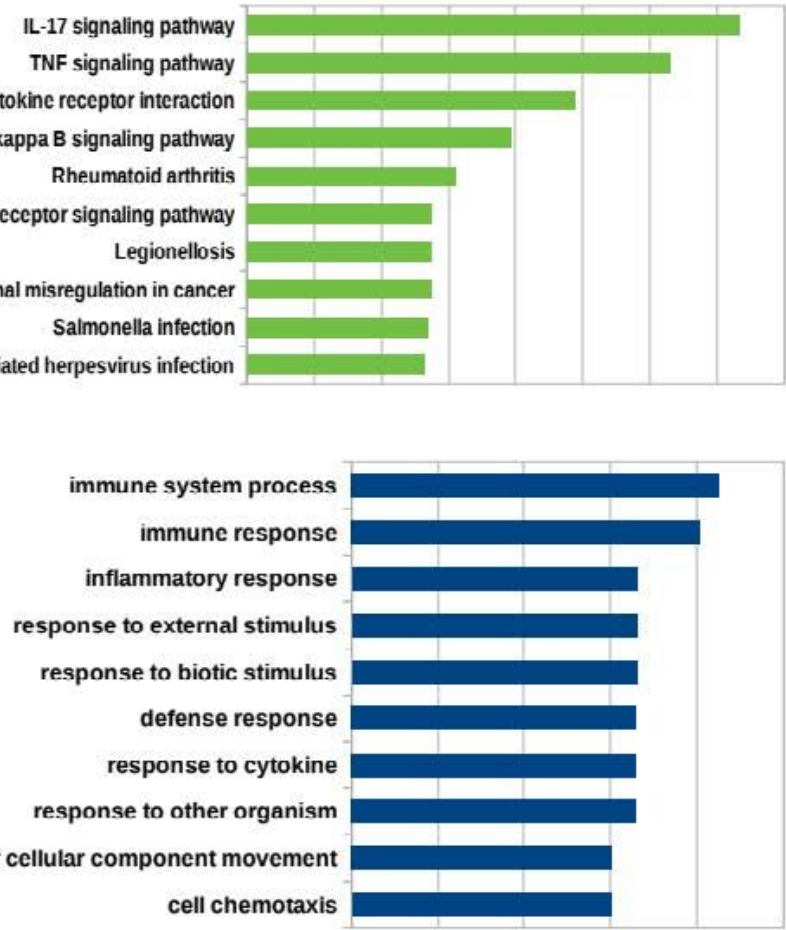

B

receptor ligand activity cytokine activity molecular function regulator protein binding cytokine receptor binding signaling receptor binding chemokine activity

CXCR chemokine receptor binding growth factor receptor binding peptidase regulator activity

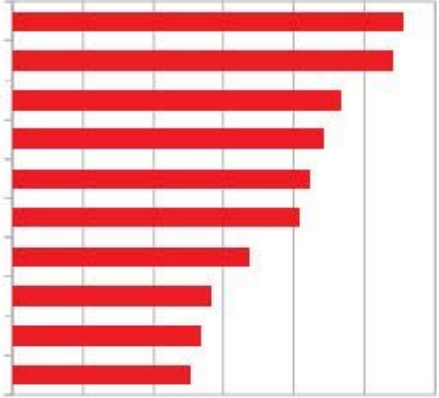

n

extracellular region extracellular space secretory granule cytoplasmic vesicle vesicle

cornified envelope cytoplasmic vesicle lumen secretory granule lumen

cytoplasm endomembrane system

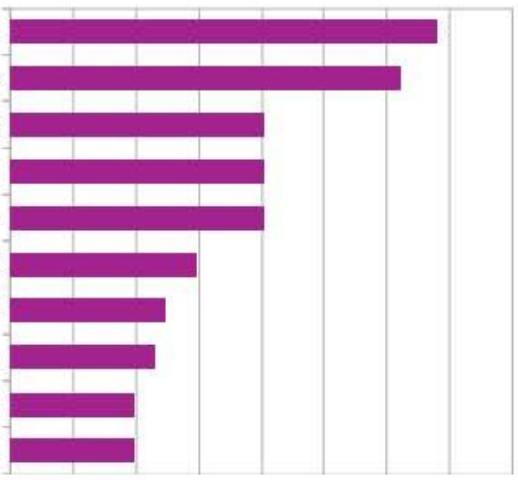

Figure 3 
Enrichment analysis of DEGs. A. KEGG pathway enrichment analysis of DEGs. B. Biological function enrichment analysis of DEGs. C. Biological process enrichment analysis of DEGs. D. Biological component enrichment analysis of DE.

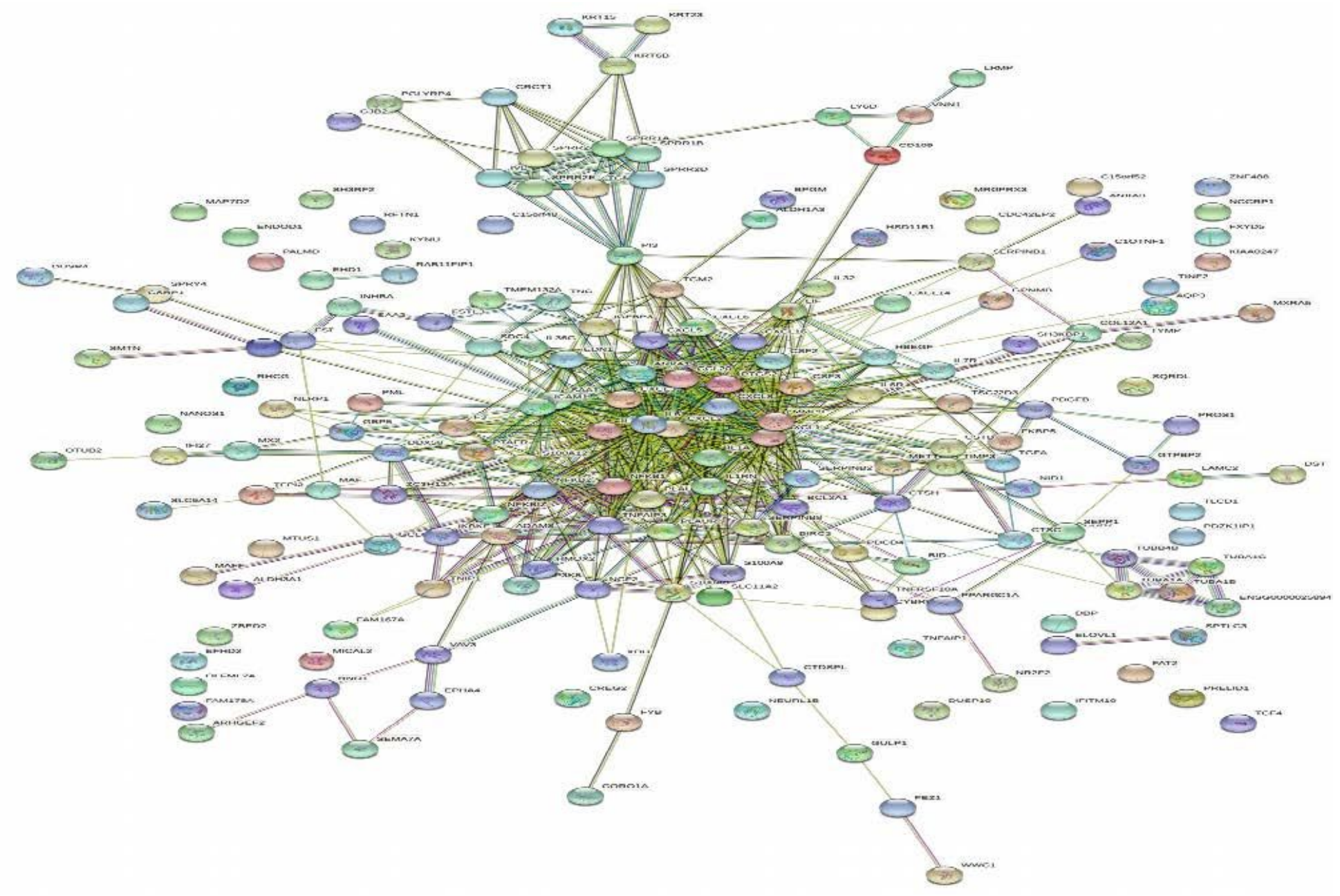

\section{Figure 4}

Unexpanded PPI network of DEGs using STRING.

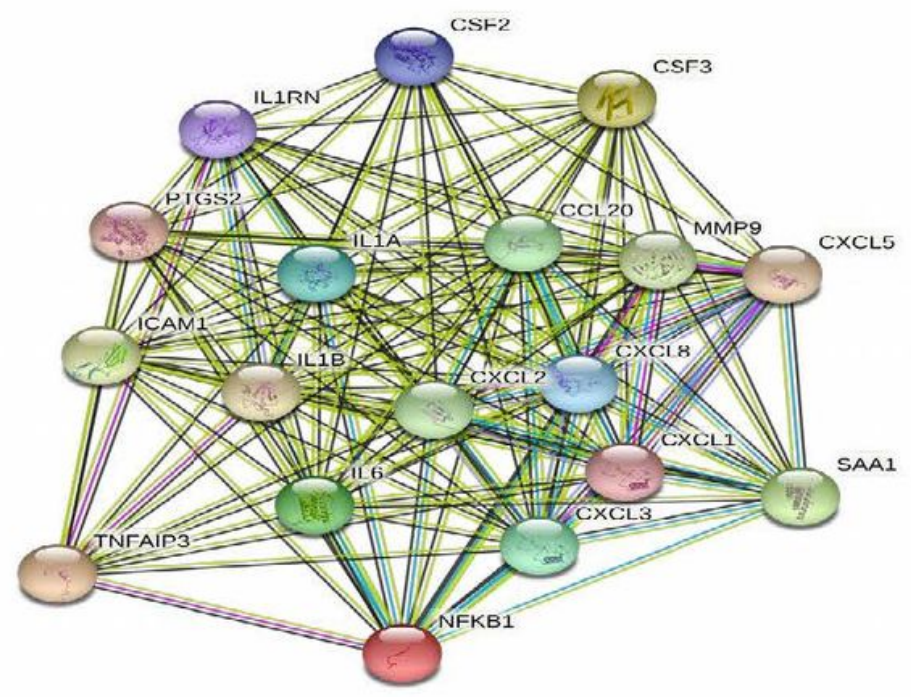


Figure 5

The PPI network of the hub genes using STRING.

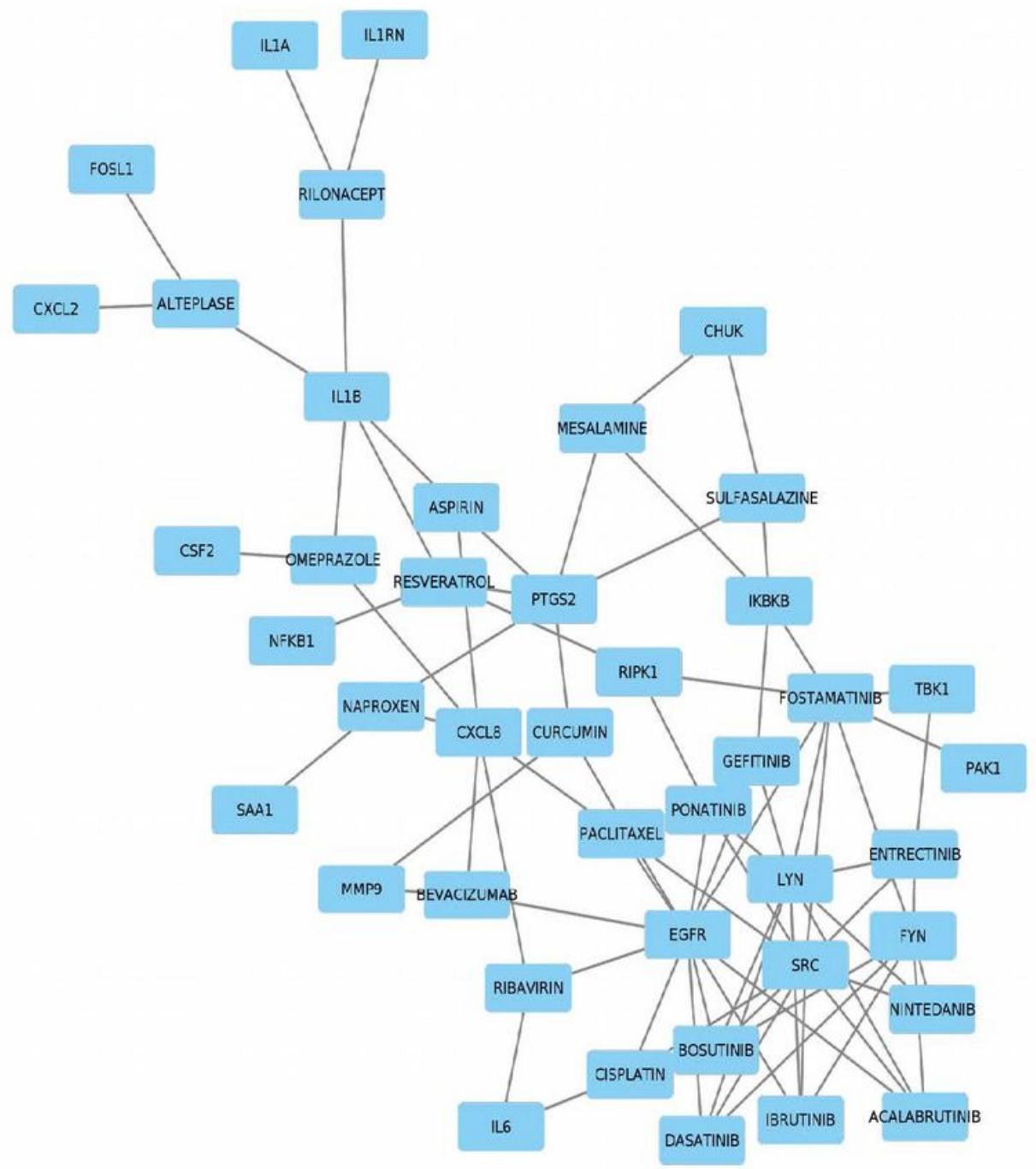

Figure 6

Drug-potential target network using Cytoscape.

\section{Supplementary Files}

This is a list of supplementary files associated with this preprint. Click to download.

- Supplementarytables.xls

- Supplementaryfigures.pdf 